\title{
Ubiquitin Signaling and Cancer Pathogenesis
}

Kaisa Haglund and Ivan Dikic

\section{1}

\section{Introduction}

Post-translational modifications of proteins allow cells to respond dynamically to intra- and extracellular stimuli to control cellular processes [1]. A modification that has been given special attention among all possible modifications is protein ubiquitination, due to the frequency of its occurrence and the key role it plays in the inducible and reversible control of signaling pathways which regulate cellular homeostasis [2-4]. Tagging of proteins with ubiquitin occurs in a three-step process through the sequential action of the ubiquitin activating (E1), conjugating (E2) and ligase (E3) enzymes [5, 6]. Ubiquitination is a dynamic and reversible modification, and the rapid removal of ubiquitin from substrates and the processing of ubiquitin chains is catalyzed by de-ubiquitinating enzymes (DUBs) [7]. The regulation of DUBs is attracting increasing interest, since they serve to switch off the ubiquitin signal or to initiate a shift between different modifications of the same lysine residue. Moreover, there seems to be an interesting interplay between E3 ubiquitin ligases and DUBs. Interactions between E3s and DUBs have been shown to regulate the stability of E3s which undergo autoubiquitination. This type of interaction also leads the DUB to its substrate and regulates the target stability [7].

Ubiquitin modification can occur in multiple ways, making it a very diverse modification with distinct cellular functions (Figure 1.1). In its simplest form, a single ubiquitin molecule is attached to a single lysine residue in a substrate, which is defined as monoubiquitination [8, 9]. Alternatively, several single ubiquitin molecules can be attached to several different lysines, which is referred to as multiple monoubiquitination or multiubiquitination [10,11]. Moreover, ubiquitin contains seven lysines itself that can be used to form various types of ubiquitin chain in an iterative process known as polyubiquitination [5, 12]. Interestingly, all seven lysines (Lys6, Lys11, Lys27, Lys29, Lys33, Lys48 and Lys63) have the potential to be used in chain formation, giving rise to chains with different linkages or branches [13].

Monoubiquitination is involved in endocytosis of plasma membrane proteins, the sorting of proteins into the multivesicular body (MVB), budding of 

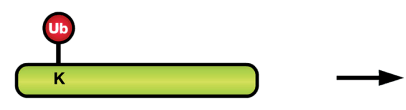

MultiUb

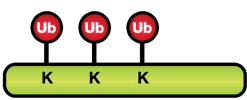

$\longrightarrow$ Endocytosis

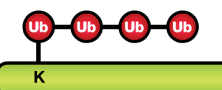

K63
Endocytosis, endosomal sorting, histone regulation, DNA repair, virus budding, nuclear export

DNA repair, endocytosis, activation of protein kinases

K48 $\rightarrow$ Proteasomal degradation

Nuclear-cytoplasmic transport, $\longrightarrow$ chromatin remodelling, DNA repair, regulation of gene transcription

\section{$\longrightarrow$ Unknown function}

Poly
SUMO
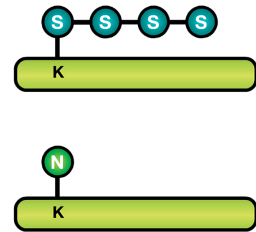

$\longrightarrow$ Control of E3 ubiquitin ligases, lysosomal receptor degradation

FAT10

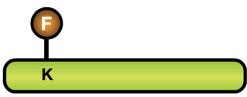
$\longrightarrow \quad \begin{aligned} & \text { Proteasomal degradation during } \\ & \text { immune response }\end{aligned}$

\section{ISG15}

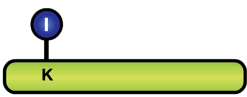

Regulation of immune response

\section{Atg8/12}

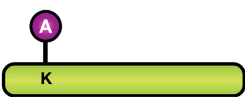

Fig. 1.1. Ubiquitin and ubiquitin-like protein (Ubl) modifications regulate a wide variety of cellular processes. Ubiquitin and Ubls share the same ubiquitin superfold and are collectively referred to as ubiquitons. All ubiquitons are attached via their C-terminal glycine residue to lysine residues in target proteins via a covalent isopeptide bond. Monoubiquitination (MonoUb) is essential for endocytosis and/or endosomal sorting of a variety of receptors, regulation of histones, DNA repair, virus budding and nuclear export. Tagging of several lysines with single ubiquitin molecules (MultiUb), is involved in endocytosis of certain RTKs and regulation of p53 localization. Polyubiquitination (PolyUb), the formation of ubiquitin chains via different lysines of ubiquitin, targets proteins for degradation in the $26 \mathrm{~S}$ proteasome when linked via lysine 48, and has non-proteolytic functions, including control of DNA repair, endocytosis and activation of protein kinases when linked via lysine 63. Sumolyation controls several processes in the cell nucleus, including DNA repair, protein localization, chromatin remodeling and gene transcription. Neddylation regulates the activity of several E3 ubiquitin ligases, including $\mathrm{Cbl}, \mathrm{Mdm} 2$ and cullins, and cooperates with ubiquitin to target EGFRs for lysosomal degradation [33, 34]. ISG15 and FAT10 are dimeric ubiquitons implicated in immune response [33, 34]. Atg8 and Atg 12 play important roles in autophagy, the degradation of bulk cytoplasmic components, by contributing to the formation of autophagosomes during nutrient starvation of cells [33, 34]. Ub, ubiquitin; K, lysine; $S$, SUMO; N, Nedd8; F, FAT10; I, ISG15; A, Atg8/12. 
retroviruses, DNA repair, histone activity and transcriptional regulation $[8,9,14$ 16]. Multiple monoubiquitination is also involved in endocytosis of receptor tyrosine kinases (RTKs) and in nuclear export of p53 [10, 11]. In the case of polyubiquitination, the functions of polyubiquitin chains linked via lysines 48 and 63 have been best characterized. Proteins that are polyubiquitinated with Lys48linked chains are recognized by ubiquitin-binding subunits of the $26 \mathrm{~S}$ proteasome and are targeted for proteasomal degradation [5, 17]. Chains linked via Lys63, on the other hand, are involved in regulating endocytosis, DNA repair and activation of NF-KB [2, 14, 18-20]. Thus, whereas Lys48-linked polybiquitination was the first proteolytic signal described, it is becoming clear that monoubiquitination and Lys63-linked polyubiquitination function in several non-proteolytic cellular processes to regulate signaling networks.

\section{1 .1}

\section{Ubiquitin Signaling Networks}

Ubiquitination is similar to phosphorylation and functions as a signaling device in cellular signaling networks. First, ubiquitination is an inducible event, which can be triggered by signals such as extracellular stimuli, phosphorylation and DNA damage [2]. This is associated with the fact that E3 ubiquitin ligases are tightly regulated by signal-induced mechanisms, such as post-translational modifications, compartmentalization, degradation and oligomerization [21, 22]. A prominent example is the ubiquitin ligase $\mathrm{Cbl}$, which is recruited to a particular phosphotyrosine residue in the epidermal growth factor receptor (EGFR) following its ligandinduced activation, and subsequently tyrosine phosphorylation of $\mathrm{Cbl}$ itself promotes its ubiquitin ligase activity and consequently ubiquitination of the EGFR [23-25].

Second, ubiquitination is a reversible signal that is modulated by the action of DUBs, which is critical for the dynamic regulation of ubiquitin networks in the cell. The regulation of DUB activity is only beginning to be understood, and structural data indicate that these enzymes are in an active conformation only when bound to ubiquitin. Some DUBs require formation of complexes with other proteins in order to become active, and it has been reported that some are inhibited by phosphorylation or degradation [7]. For example, CYLD, an important DUB in the NF-KB pathway, undergoes inhibitory phosphorylation after TNF- $\alpha$ stimulation, leading to the accumulation of one of its substrates, Lys63-ubiquitinated TRAF2 [26].

Ubiquitin mediates many of its functions by interacting with highly specialized ubiquitin-binding domains (UBDs) in downstream effector proteins. More than 15 UBDs (UBA, UIM, IUIM, UEV, GAT, CUE, PAZ, NZF, GLUE, UBM, UBZ, VHS etc.) have been discovered so far [13, 27-31]. The structures of most of these domains have been elucidated when they are complexed with ubiquitin and it appears that they have many different tertiary structures and bind ubiquitin with relatively low affinity $(50-100 \mu \mathrm{M})[13,30]$. The low affinity of UBD-ubiquitin interactions allows rapid assembly and disassembly of interaction networks, which 
facilitates dynamic biochemical processes $[9,13]$. Moreover, it is thought that a local increase in the concentration of UBD-containing proteins and UBDs, for example by the formation of multimeric complexes or the presence of several UBDs within the same protein, might increase the rate at which UBD-ubiquitin interactions occur $[9,13,30]$. Furthermore, some UBDs can bind several ubiquitin molecules simultaneously, as has been reported for the UIM of the endocytic sorting protein Hrs (hepatocyte growth factor-regulated tyrosine kinase substrate) [32]. Due to its versatility, the numerous substrates that can be tagged with ubiquitin and the various proteins containing UBDs, ubiquitination is thus involved in complex networks of interactions in time and space that regulate key cellular functions, such as signaling, endocytosis, cell cycle and DNA repair.

\section{1 .2}

\section{Ubiquitin-like Proteins}

The complexity of cellular signaling networks is further increased by modifications with ubiquitin-like (Ubl) proteins, including the small ubiquitin-related modifier (SUMO), Neural precursor cell-expressed developmentally downregulated 8 (Nedd8), interferon-stimulated gene 15 (ISG15), FAT10, Atg8 and Atg12 [33, 34], all of which regulate a variety of physiological processes (Figure 1.1). All Ubls share a similar three-dimensional structure, the ubiquitin superfold which is a $\beta$-grasp fold. Despite the varying degrees of sequence similarity, all proteins containing this fold are collectively known as ubiquitons [34].

In a manner similar to that involved in the tagging of proteins with ubiquitin, Ubls are covalently attached to their target proteins via a cascade of three enzymes (E1, E2, E3) which are partially specific for each of the Ubls [33]. As with ubiquitin, Ubls most frequently attach to lysines, although the free N-terminus can be an attachment site for both for ubiquitin and Ubls. In contrast to the ubiquitin system, Ubls generally form mono-conjugates with the substrates and not polymeric chains (Figure 1.1). SUMO conjugates have been observed, however, but their function is not yet known [35]. It is very likely that there are specialized interaction domains for all the Ubls, although they have only been described for a subset. SUMOinteracting motifs (SIMs) have been assigned [36-39], and some known UBDs interact not only with ubiquitin, but also with Nedd8 [40]. Moreover, it is interesting to note that UBDs and SIMs bind at distinct surface locations on ubiquitin and SUMO, respectively, resulting in highly specific interactions which provide some insights into the different cellular functions of these two proteins [1].

In many cases, there is an active interplay between ubiquitin and Ubls in the regulation of individual proteins and/or cellular pathways. For example, the same lysine residue can be modified with either ubiquitin or SUMO, leading to the activation of completely different downstream pathways. The modification of PCNA (proliferating cell nuclear antigen), that forms a clamp that recruits DNA polymerases to the replication fork, with either ubiquitin or SUMO induces errorprone DNA repair or DNA synthesis, respectively [14]. Moreover, there is apparent cooperation between ubiquitin and Nedd8 during downregulation of the epider- 
mal growth factor receptor (EGFR). EGF stimulation triggers Cbl-mediated neddylation of the EGFR, which in turn promotes the subsequent Cbl-mediated ubiquitination of the receptor and its degradation [40].

Further complexity in Ubl signaling networks results from the fact that Ubl domains can be found within the genetically-encoded sequence of proteins. Many proteins containing Ubl domains interact with the proteasome, but there are also several examples in which the ubiquitin fold is involved in mediating proteinprotein interactions in signal transduction cascades, consistent with the important role of ubiquitin and Ubls in both degradation and signaling pathways [34].

\section{2}

\section{Ubiquitin in Cancer Pathogenesis}

The development of cancer is a multi-step process which results from mutations in the cellular pathways that control signaling, endocytosis, cell-cycle and celldeath and interactions between the tumor and its surrounding tissue [41]. Deregulation of components of the ubiquitination machinery appears to be a common theme in the development of cancers [4, 42-44]. Mutations or overexpression of numerous E3 ubiquitin ligases can convert them to potent oncogenes and some E3s and DUBs act as tumor suppressors (Table 1.1). Several substrates that are affected by alterations in E3 and DUB activity play key roles in the cell cycle, DNA repair, NF- $\kappa \mathrm{B}$ signaling, RTK signaling and angiogenesis and their levels or activity are precisely regulated by ubiquitination (Table 1.1; Figure 1.2). In the following sections we will highlight the nature of role that the ubiquitin system plays in maintaining the homeostatic balance of these processes and why its deregulation promotes the development of different types of tumors.

\subsection{1}

\section{Ubiquitin in Cell Cycle Control}

Deregulation of cell-cycle control is a fundamental characteristic of cancer. Uncontrolled proliferation of cancer cells occurs because the precise regulation of the cell cycle has been disrupted [41]. Progression through the cell cycle is mediated by cyclin-dependent kinases (CDKs) whose activity is regulated by cyclins and CDK inhibitors (CDKIs) [43]. These undergo ubiquitin-mediated proteolysis which results in their periodic expression, ensuring that the cell cycle proceeds at normal speed. Cyclins act as accelerators of the cell cycle, whereas CDKIs function as brakes. Therefore, cyclins (D1 and E) are frequently overexpressed in human cancers and the CDKI p27 is a prominent tumor suppressor [43, 45, 46].

Three structurally-related cullin-dependent E3 ubiquitin ligases, SKP1-CUL1-Fbox-protein (SCF)/Skp2, SCF/Fbw1 and anaphase-promoting complex/cyclosome (APC/C), are involved in regulating the levels of cyclins and CDK inhibitors by promoting their polyubiquitination and degradation in the proteasome [43]. 
6 6 1 Ubiquitin Signaling and Cancer Pathogenesis

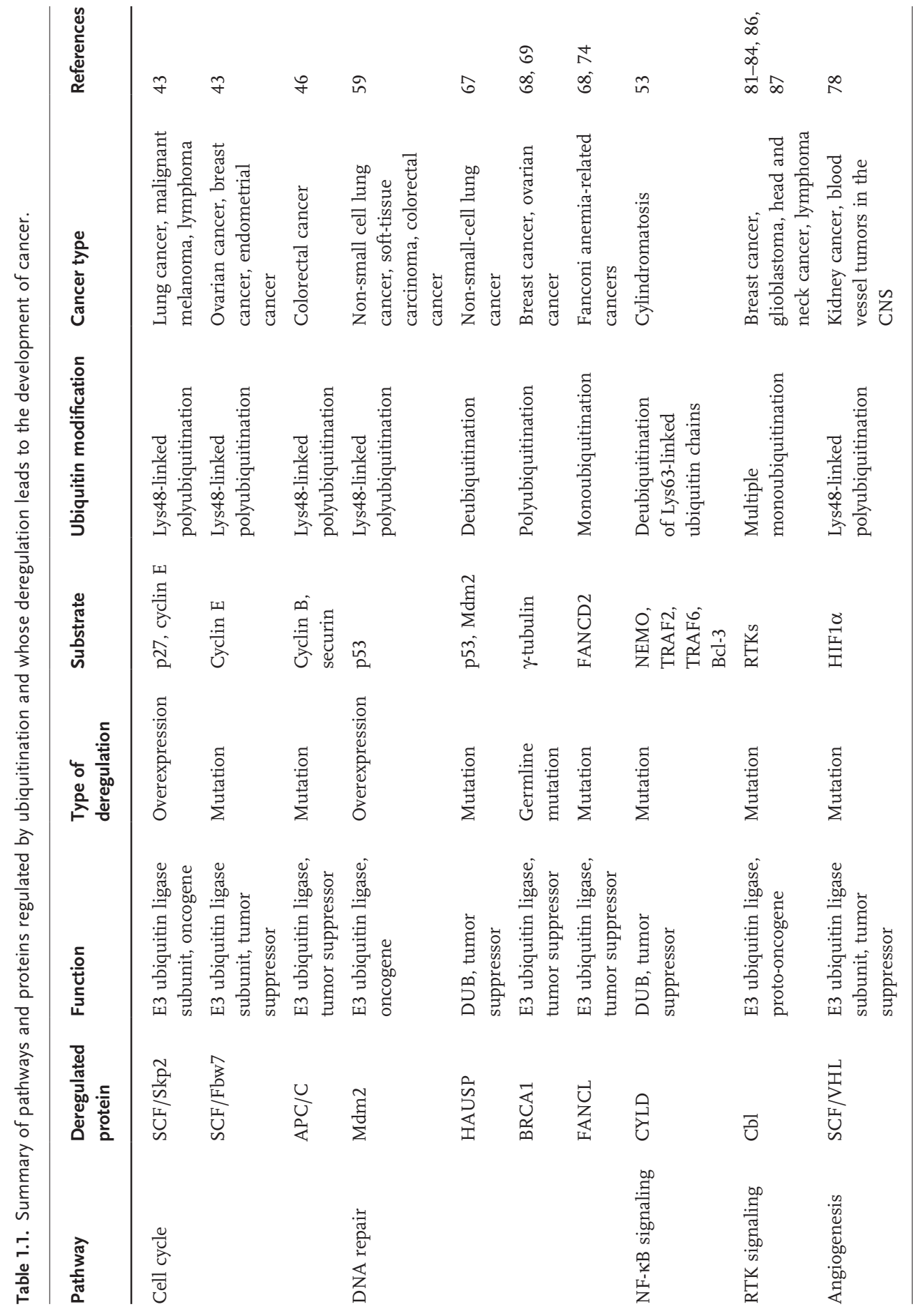




\section{A. Cell cycle}

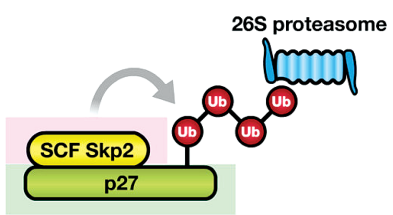

C. DNA repair

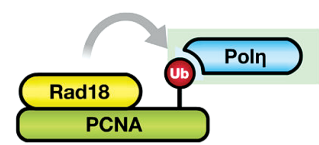

\section{E. Angiogenesis}

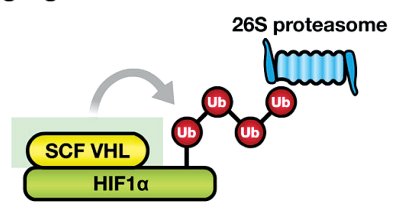

Fig. 1.2. Overview of cancer-relevant ubiquitin-dependent pathways. SCF/Skp2, Mdm2, Rad18, Cbl, SCF/VHL and TRAF6 all are E3 ubiquitin ligases (yellow) that mediate specific types of ubiquitination of their respective substrates which are indicated in the figure (p27, p53, PCNA, RTKs, HIFl $\alpha$ and NEMO) (green). The proteasome, which has UBD-containing subunits, and UBDcontaining proteins (Poln, TSG101, TAB2/3) are shown in blue. (A, B, E) Lys48-linked polyubiquitination of $\mathrm{p} 27, \mathrm{p} 53$ and $\mathrm{HIFl} \alpha$ leads to their proteasomal degradation, promoting cell cycle progression (p27, p53) or block of production of pro-angiogenic factors (HIFl $\alpha$ ). SCF/Skp2 and Mdm2 act as oncogenes, because their overexpression leads to increased proliferation and the development of cancer. SCF/VHL, on the other hand, acts as a tumor suppressor, since its mutation leads to the accumulation of $\mathrm{HIF} 1 \alpha$, aberrant angiogenesis and tumorigenesis. (C) Rad18 mediates monoubiquitination of PCNA, a modification responsible for recruiting ubiquitin binding

\section{B. DNA repair/cell cycle}

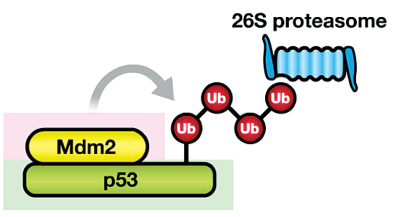

D. RTK degradation

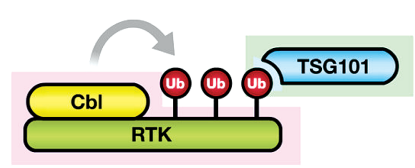

F. NF-KB signaling

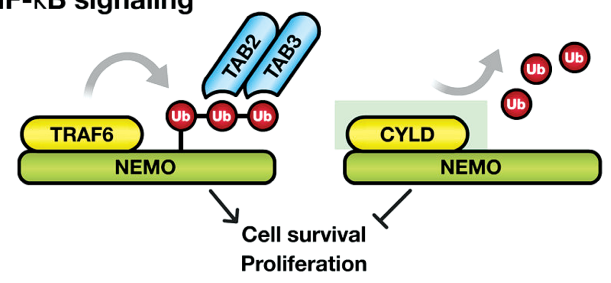

domain (UBD)-containing TLS polymerases to the site of DNA damage. Mutation of TLS polymerase Poln leads to a variant of a skin tumor syndrome called Xeroderma pigmentosum. (D) Cbl mediates multiple monoubiquitination of RTKs, which is recognized by ubiquitin-binding domains in proteins of the endocytic sorting machinery, including TSG101. Mutation of the Cbl binding site in RTKs, mutations of $\mathrm{Cbl}$ that abolish its ubiquitin ligase activity, or mutation in TSG101 all lead to defective receptor sorting and degradation, causing constitutive signaling and tumorigenesis.

(F) TRAF6 mediates Lys63-linked polyubiquitination of NEMO, which recruits the UBD-containing proteins TAB2/3, leading to activation of the protein kinase TAK 1 that is required for NF-KB activation. CYLD, the DUB that removes Lys63-linked chains from $\mathrm{NEMO}$, is mutated in a cancer syndrome called cylindromatosis. Tumor suppressors are indicated in turquoise and oncogenes or proto-oncogenes in pink. 
SCF/Skp2 targets among others p27 and cyclin E, and SCF/Fbw1 targets cyclin E for polyubiquitination and proteasomal degradation, events that regulate the G1-S transition (Figure 1.2) [47]. APC/C, on the other hand, promotes polyubiquitination and degradation of mitotic cyclins and securin, which are required for termination of the mitotic cycle and separation of the sister chromatids, respectively [46]. In this way APC/C maintains the normal chromosome number, alterations of which are a prevalent form of genetic instability in human cancers. These E3 ubiquitin ligases thus act at different time points during the cell cycle and importantly they appear to interplay in a regulatory loop [43].

Due to their central function in cell cycle progression, aberrant expression or mutations of SCF/Skp2, Fbw1 or APC/C have been found in several human cancers (Table 1) [43, 45, 46]. Skp2 has oncogenic properties in transgenic mouse models, is frequently overexpressed in lung cancers and its overexpression is correlated with poor prognosis in a wide range of cancer types [43]. Fbw1, on the other hand, acts as a tumor suppressor. Mutations in the FBW1 gene have been reported in ovarian, breast and endometrial cancer, often correlated with increased cyclin E levels [43]. APC/C also functions as a tumor suppressor and is mutated in more than $70 \%$ of colorectal carcinomas [46]. Thus, cumulative evidence indicates that deregulation of the ubiquitin system in cell-cycle control is closely linked to the development of cancer.

\section{2 .2}

\section{Ubiquitin in the NF-KB Pathway}

The NF-אB family of transcription factors triggers the expression of genes that are central mediators of cell survival, proliferation, and innate and adaptive immune responses. The role of NF- $\mathrm{KB}$ in cancer is connected to its constitutive activation of anti-apoptotic signals in both pre-neoplastic and malignant cells, and its emerging role in regulating tumor angiogenesis and invasion [48]. NF- $\mathrm{\kappa B}$ activation is controlled by ubiquitination of several of the components of the NF- $\mathrm{BB}$ pathway $[2,18,49]$. A key step in the activation of NF- $\kappa B$ is its release from the inhibitor $\mathrm{I} \kappa \mathrm{B}$ and its subsequent translocation from the cytoplasm to the nucleus where it triggers the expression of its target genes. A central regulator of this process is the IKB kinase (IKK) complex, which consists of two catalytic subunits (IKK $\alpha$ and IKK $\beta$ ) and a regulatory subunit (IKK $\gamma / \mathrm{NEMO}$ ). IKK promotes IKB phosphorylation which recruits the E3 ubiquitin ligase SCF- $\beta$ TRCP to IKB which in turn promotes Lys48-linked ubiquitination and proteasomal degradation, thereby releasing NF$\kappa \mathrm{B}[18,49]$.

Another type of ubiquitin modification is exemplified by Lys63-linked polyubiquitination which also plays a central role in NF- $\mathrm{KB}$ activation by activating protein kinases. Both IKK and the kinase that activates IKK, TGF $\beta$-activated kinase (TAK1), require Lys63-linked chains synthesized by the E3 ubiquitin ligase TNF receptor associated factor 6 (TRAF6) for their activation [18]. IKK activation requires the modification of the regulatory subunit NEMO with Lys63-linked chains [50]. TAK1 activation depends on the interaction between the UBDs of the TAK1-binding 
proteins TAB1, 2 and 3 with substrates modified with Lys63-linked polyubiquitin chains, and ubiquitinated NEMO is a likely interaction partner (Figure 1.2) [51].

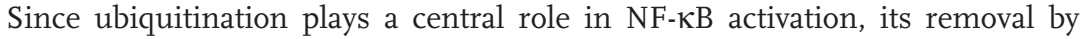
DUBs is critical to the downregulation of the NF- $\kappa B$ signal. To date, two DUBs have been identified to have important roles in regulating the NF- $\kappa \mathrm{B}$ pathway, A20 and cylindromatosis (CYLD). A20 has a dual role in downregulating NF- $\kappa B$ signaling. First, A20 specifically removes Lys63-linked ubiquitin chains from the receptorinteracting protein (RIP), an essential mediator of TNF receptor 1 (TNFR1) signaling, and subsequently it attaches Lys48-linked ubiquitin chains to promote its proteasomal degradation [52]. Whether there is a genetic link between A20 and the risk of cancer still needs to be established [4].

CYLD was originally identified as a tumor suppressor gene that is mutated in familial cylindromatosis, an autosomal dominant disease characterized by multiple tumors of the skin appendages [53]. CYLD contains a ubiquitin C-terminal hydrolase (UCH) domain and acts as a DUB that removes Lys63-linked chains from several NF- $\kappa$ B pathway members, including the ubiquitin ligases TRAF2 and TRAF6, the IKK subunit NEMO and the transcriptional co-activator Bcl-3 (Figure

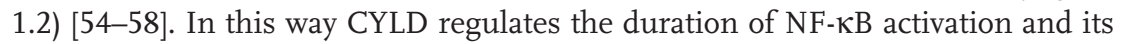
loss thus correlates with tumorigenesis.

These examples illustrate that modification of pathway components containing Lys63-linked ubiquitin chains (NEMO, TRAFs, RIP, Bcl-3) triggers the activation of NF- $\mathrm{KB}$, whereas ubiquitin removal is a common theme in its inactivation, thereby preventing excessive cell proliferation and tumor development.

\section{2 .3}

\section{Ubiquitin as a Signal in DNA Repair}

The maintenance of DNA integrity is pivotal to the prevention of cancer-promoting mutations in the genome. Cells have therefore developed elaborate DNA repair systems to respond to DNA damage. Emerging data show that ubiquitin modification plays a major role in DNA repair response both by regulating cell cycle arrest (p53, Mdm2, HAUSP, BRCA1 and FANCD2) and by controlling trans-lesion DNA synthesis (PCNA and TLS polymerases).

\subsubsection{1 p53 Pathway}

The $p 53$ gene is mutated in more than $50 \%$ of human cancers. p53 is a transcription factor with an essential role in promoting cell-cycle arrest, apoptosis and DNA repair when cells encounter DNA damage. In this way, p53 hinders proliferation of damaged cells and acts as a tumor suppressor [59, 60]. In order to maintain cellular homeostasis, the levels of p53 are highly regulated in cells. In unstressed cells, the levels of p53 are kept low and this is mediated by ubiquitin-dependent proteasomal degradation. Mdm2 is a RING-type E3 ubiquitin ligase responsible for promoting both monoubiquitination and Lys48-linked polyubiquitination of p53 in a dose-dependent manner (Figure 1.2) [11]. Monoubiquitination of p53 promotes its nuclear export and polyubiquitination, its degradation by nuclear 
proteasomes. Normally, the interaction between Mdm2 and p53 is disrupted when cells encounter DNA damage or other stresses, promoting an accumulation of p53 in the nucleus, cell-cycle arrest and DNA repair [61]. Overexpression of Mdm2, on the other hand, leads to aberrant deactivation of p53, which is observed in many types of tumors (Table 1.1) [59, 62, 63].

Herpes simplex-associated ubiquitin-specific protease (HAUSP) is involved in p53 deubiquitination and stabilization $[64,65]$. Importantly, its overexpression is sufficient to promote cell-cycle arrest and apoptosis, suggesting that it could act as a tumor suppressor [65]. On the other hand, disruption of the HAUSP gene in human cancer cell lines by targeted homologous recombination, also leads to p53 stabilization and activation [66]. These contradictory results could be explained by the presence of other targets of HAUSP, such as Mdm2, which determine p53 levels [64]. Nevertheless, mutations of the HAUSP gene are associated with an increased risk for non-small-cell lung cancer [67].

\subsubsection{BRCA1 and FANCD2}

The breast cancer susceptibility genes $B R C A 1$ and $B R C A 2$ and products of the Fanconi anemia (FA) gene act as tumor suppressors. They function in a network of interconnected biological processes and have important roles in cell-cycle checkpoint control and DNA repair of double strand breaks by mediating homologous recombination [60]. Germline mutations in one allele of either BRCA1 or BRCA2 cause hereditary breast and ovarian cancer syndrome and mutations in FA genes (FANCA, FANCB, FANCC, FANCD1, FANCD2 etc.) can cause FA, a genetic disorder associated with increased susceptibility to cancer [68].

BRCA1 acts as a RING-type E3 ubiquitin ligase and its activity is increased when it is complexed with the structurally and functionally related BRCA1-associated RING domain 1 (BARD1) ubiquitin ligase [69]. Specific mutations in the RING domain of BRCA1 abolish its ubiquitin ligase activity and tumor suppression capabilities. Interestingly, BRCA1 and BARD1 preferentially promote formation of Lys6-linked chains, a chain type that seems to be primarily involved in substrate stabilization [69].

$B R C A 1$ - and BARD1-deficient mice show centrosome amplification, defective G2-M checkpoint control and genetic instability [69]. Among the ubiquitinated targets of BRCA1/BARD1 is the centrosome component $\gamma$-tubulin [70]. Following their duplication during cell division, centrosomes help to form the spindle apparatus that segregates the duplicated chromosomes into daughter cells. Mutation of the ubiquitination site in $\gamma$-tubulin leads to amplification of centrosome numbers, a defect associated with chromosome missegregation and the development of cancer [70].

When DNA is damaged, BRCA1 binds to FANCD2 in nuclear foci that are required for cell-cycle checkpoint control and DNA repair [71]. The localization of FANCD2 to these foci is promoted by its monoubiquitination, suggesting that monoubiquitin-binding proteins might be involved in its recruitment [72]. FANCD2 undergoes monoubiquitination in BRCA1-/- cells, indicating that another E3 ubiquitin ligase promotes this modification [73]. Indeed, a component 
of the nuclear FA-protein complex, FANCL, possesses E3 ubiquitin ligase activity against FANCD2 via its RING-finger-like plant domain (PHD) [74]. Deubiquitination of FANCD2 by ubiquitin-specific protease 1 (USP1), on the other hand, may play an important role when cells restart the cell cycle after DNA damage [75].

\subsubsection{PCNA and TLS Polymerases}

DNA damage blocks the progression of the replication fork and in order to avoid stalling the replication process and circumventing the damaged sites, cells replace the high-fidelity replicative polymerase Pol $\delta$ with one of the five specialized low stringency DNA polymerases which are able to perform trans-lesion DNA synthesis (TLS) across different types of damage [76]. That TLS is crucial for cells is emphasized by the fact that defects in TLS polymerases can cause disease. Mutations in TLS polymerase Poln are found in patients suffering from a variant of Xeroderma pigmentosum, a UV-induced skin tumor syndrome [77].

Proliferating cell nuclear antigen (PCNA) functions to recruit different polymerases to the site of DNA replication or repair, and its ubiquitination and deubiquitination plays a major role in the polymerase switch. Non-ubiquitinated PCNA recruits the replicative polymerase Pol $\delta$ during DNA replication. Certain types of DNA damage, on the other hand, induce Rad18-mediated monoubiquitination of PCNA. This modification triggers the recruitment of TLS polymerases, all of which contain UBDs, the so-called ubiquitin-binding motif (UBM) or ubiquitin-binding zinc finger (UBZ) (Figure 1.2) [14, 27]. After trans-lesion synthesis has taken place, the low fidelity TLS polymerases are exchanged for Pol $\delta$ to ensure accurate continued replication. Therefore, the DUB USP1 removes ubiquitin from PCNA during normal replication to allow recruitment of Pol $\delta$ and is degraded once the DNA becomes damaged, again allowing monoubiquitination of PCNA and recruitment of the TLS polymerases [20].

\subsection{4}

\section{Ubiquitin Networks in Angiogenesis}

Rapidly growing tumors require efficient blood and nutrient supply and therefore secrete growth factors, such as vascular endothelial growth factor (VEGF) and platelet derived growth factor (PDGF), to promote angiogenesis, the formation of new capillaries. Therefore, it is not surprising that an anti-angiogenic protein, such as VHL (von Hippel-Lindau), would be a tumor suppressor [78]. The VHL gene encodes a component of an SCF-like ubiquitin ligase and is mutated in patients suffering from the familial cancer susceptibility, von Hippel-Lindau syndrome, that is associated with cancer of the kidney and tumors in the blood vessels of the central nervous system [78, 79]. Under normoxic conditions, VHL binds to the hydroxylated $\alpha$-subunits of the hypoxia-inducible factor (HIF) heterodimeric transcription factors and targets them for polyubiquitination and proteasomal degradation (Figure 1.2) [80]. During hypoxic conditions, HIF1 $\alpha$ is not hydroxylated and can thus not be bound by VHL, leading to its stabilization. HIF1 $\alpha$ then triggers 
the transcription of several genes encoding pro-angiogenic growth factors, including VEGF, PDGF $\beta$ and transforming growth factor $\alpha$ (TGF $\alpha$ ) [78]. Mutation of VHL is thought to lead to constantly increased levels of HIF1 $\alpha$ and its target growth factors even under normoxic conditions [78], thus stimulating the formation of new blood vessels and tumors.

\section{2 .5}

\section{Ubiquitin Networks in Receptor Endocytosis}

Constitutive receptor tyrosine kinase (RTK) signaling, resulting from receptor overexpression, autocrine growth factor loops and activating mutations, can cause cell transformation and cancer [81]. Moreover, loss of negative regulation of RTKs is an important factor contributing to enhanced receptor signaling [82-84]. RTKs are downregulated by endocytosis and lysosomal degradation, which requires ligand-induced Cbl-mediated receptor multiple monoubiquitination, Lys63-linked polyubiquitination and neddylation (Figure 1.2) [9, 10, 40, 85]. Ubiquitin attached to RTKs serves as a sorting tag that is recognized by UBD-containing endocytic proteins along the endocytic pathway, ensuring that they targeted into the inner vesicles of the multivesicular body (MVB), which destines them for lysosomal degradation $[9,15,16]$. Therefore, RTK mutations that lead to the loss of the binding site for the ubiquitin ligase $\mathrm{Cbl}$ in addition to $\mathrm{Cbl}$ mutants lacking ubiquitin ligase activity, cause defective downregulation of the receptor [83, 84]. Prominent examples of RTKs that have been found mutated in tumors and have escaped Cbl-mediated ubiquitination and degradation include EGFR (EGFRvV, v-erbB and EGFRvIII), MET (TRP-MET) and c-Kit (v-Kit) [83, 86, 87]. Oncogenic forms of Cbl (v-Cbl, Cbl-70Z, $\Delta \mathrm{Y} 368-\mathrm{Cbl}, \Delta \mathrm{Y} 371-\mathrm{Cbl}$ ) all lack ubiquitin ligase activity and are thought to act as dominant negative proteins and to compete with endogenous $\mathrm{Cbl}$ for binding to activated RTKs [24, 25]. Deletions of the extracellular area of the EGFR (EGFRvIII) are found in approximately $40 \%$ of glioblastomas and the EGFR family member ErbB2 is frequently overexpressed in breast cancer [87]. Overexpression of ErbB2 favors the formation of EGFR/ErbB2 heterodimers which recruit $\mathrm{Cbl}$ less efficiently, and are thus not degraded, but rather recycled back to the cell surface [88-90].

Interestingly, components of the endosomal sorting complex required for transport (ESCRT) machinery that sorts ubiquitinated cargo into the MVB [15, 91], are also linked to the development of tumors. Mutations in the components of the ESCRT-I tumor susceptibility gene 101 (TSG101) and hepatocellular carcinomarelated protein 1 (HCRP1) have been implicated in tumor development [84, 92, 93]. TSG101 contains a ubiquitin-binding UEV domain that binds to ubiquitinated cargo and is required for effective receptor sorting into the MVB (Figure 1.2) [15]. Moreover, mutations of erupted (TSG101) and Vps25 (an ESCRT-II component) have been shown to cause neoplastic tumor growth in the fruit fly [94-98]. Thus, proper ubiquitin-dependent lysosomal degradation of activated RTKs prevents constitutive receptor signaling and carcinogenesis. 


\section{3}

\section{Targeting Ubiquitin Networks in Cancers}

Due to its common deregulation in the development of cancers, targeting the ubiquitin system in cancer therapeutics emerges as a promising approach. The major challenge is to develop drugs that specifically act on the desired ubiquitin system component or substrate without affecting other pathways. Possible strategies involve inhibiting ubiquitin activation or conjugation, ubiquitin ligase activity of oncogenic E3s, by blocking either E2 or substrate binding, or inhibiting the degradation of cancer-preventing tumor suppressors [99]. Since the ubiquitin activation and proteasomal degradation steps involve ATP-dependent and proteolytic enzymes, respectively, which are classical drug targets, they represent therapeutically attractive points of intervention [99]. The major concern with these strategies, however, is their wide action on numerous substrates and pathways within the cell which may produce severe side effects. Intervening in the E3-substrate interaction therefore represents a more selective approach which could lead to more effective treatment and fewer nonspecific effects (Figure 1.3).

\subsection{1}

\section{Targeting Interactions between E3s and their Substrates}

This strategy has been successfully applied when targeting the interaction between the oncogenic E3 ubiquitin ligase Mdm2 and the tumor suppressor p53 with two

\section{A. E3-substrate interaction}

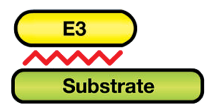

\section{Proteasome recognition}

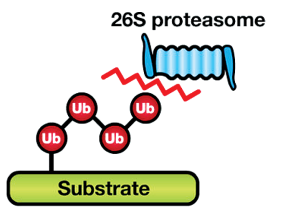

Fig. 1.3. Different approaches for targeting the ubiquitin system in cancer therapy.

(A) Interference with the interaction between the E3 ubiquitin ligase and the substrate.

Examples: Nutlin and RITA. (B) Inhibition of the catalytic activity of the proteasome.

Example: Bortezomib. (C) Interference with the interaction between Lys48polyubiquitinated substrates and the

\section{B. Proteasome inhibition}

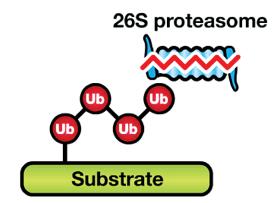

D. Ubiquitin-UBD interaction

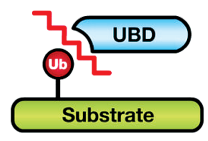

proteasome. Example: Ubistatins.

(D) Interference with the interaction between ubiquitinated substrates and the corresponding ubiquitin-binding domain (UBD)-containing proteins. The main difficulty with all these strategies is in achieving optimum specificity and selectivity. See the main text for more details. 
types of small molecule inhibitors that were identified in anti-cancer drug screens, Nutlins (cis-imidazole derivatives) and RITA (2,5-bis(5-hydroxymethyl-2thienyl)furan). Nutlins occupy the p53 binding pocket of Mdm2 and RITA binds p53 and in this way they both prevent the p53-Mdm2 interaction [99]. Consequently, both compounds stabilize p53, leading to p53-dependent cell-cycle arrest in cancer cell lines and the inhibition of tumor growth in nude mice [99]. Although the initial studies on these inhibitors seem promising, their bioavailability and usefulness in the treatment of human cancer need to be thoroughly studied. Despite higher specificity, major concerns still remain: do these interventions yield unwanted effects such as affecting other substrates of Mdm2, other interaction partners of $\mathrm{p} 53$ or $\mathrm{p} 53$-related proteins such as $\mathrm{p} 63$ and $\mathrm{p} 73$ ? These basic concerns apply in each of the cases where the interaction surface between the E3 ubiquitin ligase and the substrate is targeted.

\subsection{2}

\section{Targeting the Proteasome}

Surprisingly, the biggest success so far in targeting the ubiquitin system in cancer therapy has been the development of Bortezomib, a small molecule proteasome inhibitor that binds reversibly to the active site of the $20 \mathrm{~S}$ proteasome subunit [100]. Despite concerns regarding the lack of specificity due to the inhibition of the entire proteasomal protein degradation system, this inhibitor is being successfully used clinically in the treatment of relapsed, refractory multiple myeloma, and is being studied in a variety of hematological cancers and solid tumors, including non-Hodgkin's lymphoma, prostate, breast and non-small-cell lung cancers. Bortezomib is thought to inhibit cell proliferation by blocking the degradation of proteins involved in cell-cycle control and apoptosis (including p53, cyclins and IкB) [101]. Interestingly, Bortezomib shows selective cytotoxicity against cancer cells compared to normal cells both in vitro and in vivo [99]. Therefore, development of strategies involving proteasome inhibitors may be useful in the therapy of certain types of tumors (Figure 1.3).

\subsection{3}

\section{Other Approaches}

Apart from targeting the ubiquitin system itself, some clinically-effective monoclonal antibodies and small molecule inhibitors have been shown to promote ubiquitin-dependent degradation of oncogenic proteins. Such an example is Herceptin, a monoclonal antibody used for the treatment of breast cancer tumors overexpressing ErbB2 which increases Cbl-mediated ErbB2 ubiquitination and degradation [102].

Another promising and challenging approach to targeting the ubiquitin system in cancer therapy is to alter the ubiquitin-induced protein-protein interactions in cells [4]. Although this approach offers more specificity than any of the above-mentioned strategies due to the fact that there are more ubiquitin-induced 
interactions than existing enzymes, there are still many difficulties related to this strategy. The main obstacles include targeting the flat and hydrophobic interaction surface between ubiquitin and UBDs and dealing with the low affinities of such interactions. Modulators of polyubiquitin chain recognition, the ubistatins, have been shown to bind specifically to the interfaces between Lys48-linked ubiquitin molecules, and to inhibit ubiquitin-dependent proteasomal degradation of certain substrates (Figure 1.3) [103]. The ubistatins are however not cell-permeable, but it may be possible to use them to target interaction surfaces between ubiquitin and UBDs after further developing them and increasing their bioavailability. Other types of inhibitor of ubiquitin-UBD interactions could target either the hydrophobic surface of ubiquitin containing Ile44, with which most UBDs interact, or specific UBDs (Figure 1.3). However, these approaches are also associated with issues of specificity, since ubiquitin and ubiquitin chains are attached to numerous proteins and UBDs are found in a vast variety of proteins. Despite these drawbacks, the increasing interest and knowledge gained in this field will ensure that several novel strategies for targeting the ubiquitin system with higher specificity will be developed in the near future.

\section{4}

\section{Conclusions and Future Perspectives}

In conclusion, we note that there is a strong link between alterations in ubiquitin signaling networks and the hallmarks of cancer, including uncontrolled proliferation and cell cycle divisions (SCF ligases, NF- $\mathrm{\kappa B}$ ), increased cellular signaling (RTKs, NF-KB), defective endocytosis (Cbl, RTKs, TSG101), increased cell survival (NF- $\kappa \mathrm{B})$, defective DNA repair (p53, BRCA1, TLS polymerases) and increased angiogenesis (VHL). A complete understanding of the interplay between ubiquitination and other post-translational modifications such as phosphorylation and acetylation, between ubiquitin ligases and DUBs, as well as ubiquitin and Ubls in cellular networks will have a great impact on our insight into cancerpromoting mechanisms and our ability to design smart drugs for the treatment of cancer.

\section{References}

1 Seet, B.T., Dikic, I., Zhou, M.M. and Pawson, T. (2006) Reading protein modifications with interaction domains. Nat Rev Mol Cell Biol 7, 473-483.

2 Haglund, K. and Dikic, I. (2005) Ubiquitylation and cell signalling. EMBO J 24, 3353-3359.

3 Sigismund, S., Polo, S. and Di Fiore, P.P. (2004) Signaling through monoubiquitination. Curr Top Microbiol Immunol 286, 149-185.

4 Hoeller, D., Hecker, C.M. and Dikic, I. (2006) Ubiquitin and ubiquitin-like proteins in cancer pathogenesis. Nat Rev Cancer 6, 776-788.

5 Hershko, A. and Ciechanover, A. (1998) The ubiquitin system. Annu Rev Biochem 67, 425-479. 
6 Pickart, C.M. and Eddins, M.J. (2004) Ubiquitin: structures, functions, mechanisms. Biochim Biophys Acta 1695, 55-72.

7 Nijman, S.M., Luna-Vargas, M.P., Velds, A., Brummelkamp, T.R., Dirac, A.M., Sixma, T.K. and Bernards, R. (2005) A genomic and functional inventory of deubiquitinating enzymes. Cell 123, 773-786.

8 Hicke, L. (2001) Protein regulation by monoubiquitin. Nat Rev Mol Cell Biol 2, 195-201.

9 Haglund, K., Di Fiore, P.P. and Dikic, I. (2003) Distinct monoubiquitin signals in receptor endocytosis. Trends Biochem Sci 28, 598-603.

10 Haglund, K., Sigismund, S., Polo, S., Szymkiewicz, I., Di Fiore, P.P. and Dikic, I. (2003) Multiple monoubiquitination of RTKs is sufficient for their endocytosis and degradation. Nat Cell Biol 5, 461-466.

11 Li, M., Brooks, C.L., Wu-Baer, F., Chen, D., Baer, R. and Gu, W. (2003) Monoversus polyubiquitination: differential control of $\mathrm{p} 53$ fate by Mdm2. Science 302, 1972-1975.

12 Pickart, C.M. and Fushman, D. (2004) Polyubiquitin chains: polymeric protein signals. Curr Opin Chem Biol 8, 610-616.

13 Hicke, L., Schubert, H.L. and Hill, C.P. (2005) Ubiquitin-binding domains. Nat Rev Mol Cell Biol 6, 610-621.

14 Hoege, C., Pfander, B., Moldovan, G.L., Pyrowolakis, G. and Jentsch, S. (2002) RAD6-dependent DNA repair is linked to modification of PCNA by ubiquitin and SUMO. Nature 419, 135-141.

15 Katzmann, D.J., Odorizzi, G. and Emr, S.D. (2002) Receptor downregulation and multivesicular-body sorting. Nat Rev Mol Cell Biol 3, 893-905.

16 Hicke, L. and Dunn, R. (2003) Regulation of membrane protein transport by ubiquitin and ubiquitinbinding proteins. Annu Rev Cell Dev Biol 19, 141-172.

17 Pickart, C. and Cohen, R.E. (2004) Proteasomes and their kin: proteases in the machine age. Nat Rev Mol Cell Biol 5, 177-187.
18 Krappmann, D. and Scheidereit, C. (2005) A pervasive role of ubiquitin conjugation in activation and termination of IkappaB kinase pathways. EMBO Rep 6, 321-326.

19 Galan, J.M. and Haguenauer-Tsapis, R. (1997) Ubiquitin lys63 is involved in ubiquitination of a yeast plasma membrane protein. EMBO J 16, 5847-5854.

20 Huang, T.T., Nijman, S.M., Mirchandani, K.D., Galardy, P.J., Cohn, M.A., Haas, W., Gygi, S.P., Ploegh, H.L., Bernards, R. and D'Andrea, A.D. (2006) Regulation of monoubiquitinated PCNA by DUB autocleavage. Nat Cell Biol 8, 339-347.

21 D'Azzo, A., Bongiovanni, A. and Nastasi, T. (2005) E3 ubiquitin ligases as regulators of membrane protein trafficking and degradation. Traffic $\mathbf{6}$, 429-441.

22 Gao, M. and Karin, M. (2005) Regulating the regulators: control of protein ubiquitination and ubiquitin-like modifications by extracellular stimuli. Mol Cell 19, 581-593.

23 Levkowitz, G., Waterman, H., Ettenberg, S.A., Katz, M., Tsygankov, A.Y., Alroy, I., Lavi, S., Iwai, K., Reiss, Y., Ciechanover, A., et al. (1999) Ubiquitin ligase activity and tyrosine phosphorylation underlie suppression of growth factor signaling by c-Cbl/Sli-1. Mol Cell 4, 1029-1040.

24 Thien, C.B. and Langdon, W.Y. (2001) Cbl: many adaptations to regulate protein tyrosine kinases. Nat Rev Mol Cell Biol 2, 294-307.

25 Schmidt, M.H. and Dikic, I. (2005) The $\mathrm{Cbl}$ interactome and its functions. Nat Rev Mol Cell Biol 6, 907-919.

26 Reiley, W., Zhang, M., Wu, X., Granger, E. and Sun, S.C. (2005) Regulation of the deubiquitinating enzyme CYLD by IkappaB kinase gamma-dependent phosphorylation. Mol Cell Biol 25, 3886-3895.

27 Bienko, M., Green, C., Crosetto, N., Rudolf, F., Zapart, G., Coull, B., Kannouche, P., Wider, G., Peter, M., Lehmann, A.R., et al. (2005) Ubiquitinbinding domains in Y-family polymerases regulate tranlesion synthesis. Science 310, 1821-1824. 
28 Lee, S., Tsai, Y.C., Mattera, R., Smith, W.J., Kostelansky, M.S., Weissman, A.M., Bonifacino, J.S. and Hurley, J.H. (2006) Structural basis for ubiquitin recognition and autoubiquitination by Rabex-5. Nat Struct Mol Biol 13, 264-271.

29 Penengo, L., Mapelli, M., Murachelli, A.G., Confalonieri, S., Magri, L., Musacchio, A., Di Fiore, P.P., Polo, S. and Schneider, T.R. (2006) Crystal structure of the ubiquitin binding domains of rabex-5 reveals two modes of interaction with ubiquitin. Cell 124, 1183-1195.

30 Harper, J.W. and Schulman, B.A. (2006) Structural complexity in ubiquitin recognition. Cell 124, 1133-1136.

31 Slagsvold, T., Aasland, R., Hirano, S., Bache, K.G., Raiborg, C., Trambaiolo, D., Wakatsuki, S. and Stenmark, H. (2005) Eap45 in mammalian ESCRT-II binds ubiquitin via a phosphoinositideinteracting GLUE domain. J Biol Chem 280, 19600-19606.

32 Hirano, S., Kawasaki, M., Ura, H., Kato, R., Raiborg, C., Stenmark, H. and Wakatsuki, S. (2006) Double-sided ubiquitin binding of Hrs-UIM in endosomal protein sorting. Nat Struct Mol Biol 13, 272-277.

33 Kerscher, O., Felberbaum, R. and Hochstrasser, M. (2006) Modification of proteins by ubiquitin and ubiquitin-like proteins. Annu Rev Cell Dev Biol 22, 159-180.

34 Welchman, R.L., Gordon, C. and Mayer, R.J. (2005) Ubiquitin and ubiquitin-like proteins as multifunctional signals. Nat Rev Mol Cell Biol 6, 599-609.

35 Bylebyl, G., Belichenko, I. and Johnson, E. (2003) The SUMO isopeptidase Ulp2 prevents accumulation of SUMO chains in yeast. J Biol Chem 278, 44113-44120.

36 Hecker, C.M., Rabiller, M., Haglund, K., Bayer, P. and Dikic, I. (2006) Specification of SUMO1- and SUMO2interacting motifs. J Biol Chem 281, 16117-16127.

37 Hannich, J.T., Lewis, A., Kroetz, M.B., Li, S.J., Heide, H., Emili, A. and Hochstrasser, M. (2005) Defining the
SUMO-modified proteome by multiple approaches in Saccharomyces cerevisiae. J Biol Chem 280, 4102-4110.

38 Song, J., Durrin, L.K., Wilkinson, T.A., Krontiris, T.G. and Chen, Y. (2004) Identification of a SUMO-binding motif that recognizes SUMO-modified proteins. Proc Natl Acad Sci U S A 101, 14373-14378.

39 Minty, A., Dumont, X., Kaghad, M. and Caput, D. (2000) Covalent modification of p73alpha by SUMO-1. Two-hybrid screening with p73 identifies novel SUMO-1-interacting proteins and a SUMO-1 interaction motif. $J$ Biol Chem 275, 36316-36323.

40 Oved, S., Mosesson, Y., Zwang, Y., Santonico, E., Shtiegman, K., Marmor, M.D., Kochupurakkal, B.S., Katz, M., Lavi, S., Cesareni, G. and Yarden, Y. (2006) Conjugation to Nedd8 instigates ubiquitylation and down-regulation of activated receptor tyrosine kinases. J Biol Chem 281, 21640-21651.

41 Hanahan, D. and Weinberg, R.A. (2000) The hallmarks of cancer. Cell 100, 5770.

42 Fang, S., Lorick, K.L., Jensen, J.P. and Weissman, A.M. (2003) RING finger ubiquitin protein ligases: implications for tumorigenesis, metastasis and for molecular targets in cancer. Semin Cancer Biol 13, 5-14.

43 Nakayama, K.I. and Nakayama, K. (2006) Ubiquitin ligases: cell-cycle control and cancer. Nat Rev Cancer 6, 369-381.

44 Jiang, Y.H. and Beaudet, A.L. (2004) Human disorders of ubiquitination and proteasomal degradation. Curr Opin Pediatr 16, 419-426.

45 Mani, A. and Gelmann, E.P. (2005) The ubiquitin-proteasome pathway and its role in cancer. J Clin Oncol 23, 4776-4789.

46 Wasch, R. and Engelbert, D. (2005) Anaphase-promoting complex-dependent proteolysis of cell cycle regulators and genomic instability of cancer cells. Oncogene 24, 1-10.

47 Guardavaccaro, D. and Pagano, M. (2004) Oncogenic aberrations of cullindependent ubiquitin ligases. Oncogene 23, 2037-2049. 
48 Karin, M. (2006) Nuclear factor-kappaB in cancer development and progression. Nature 441, 431-46.

49 Karin, M. and Ben-Neriah, Y. (2000) Phosphorylation meets ubiquitination: the control of NF-[kappa]B activity. Annu Rev Immunol 18, 621-663.

50 Zhou, H., Wertz, I., O'Rourke, K., Ultsch, M., Seshagiri, S., Eby, M., Xiao, W. and Dixit, V.M. (2004) Bcl10 activates the NF-kappaB pathway through ubiquitination of NEMO. Nature 427, 167-171.

51 Kanayama, A., Seth, R.B., Sun, L., Ea, C.K., Hong, M., Shaito, A., Chiu, Y.H., Deng, L. and Chen, Z.J. (2004) TAB2 and TAB3 activate the NF-kappaB pathway through binding to polyubiquitin chains. Mol Cell 15, 535-548.

52 Wertz, I.E., O’Rourke, K.M., Zhou, H., Eby, M., Aravind, L., Seshagiri, S., Wu, P., Wiesmann, C., Baker, R., Boone, D.L. et al. (2004) De-ubiquitination and ubiquitin ligase domains of A20 downregulate NF-kappaB signalling. Nature 430, 694-699.

53 Bignell, G.R., Warren, W., Seal, S., Takahashi, M., Rapley, E., Barfoot, R., Green, H., Brown, C., Biggs, P.J., Lakhani, S.R., et al. (2000) Identification of the familial cylindromatosis tumoursuppressor gene. Nat Genet 25, 160-165.

54 Trompouki, E., Hatzivassiliou, E., Tsichritzis, T., Farmer, H., Ashworth, A. and Mosialos, G. (2003) CYLD is a deubiquitinating enzyme that negatively regulates NF-kappaB activation by TNFR family members. Nature 424, 793-796.

55 Brummelkamp, T.R., Nijman, S.M., Dirac, A.M. and Bernards, R. (2003) Loss of the cylindromatosis tumour suppressor inhibits apoptosis by activating NF-kappaB. Nature 424, 797-801.

56 Kovalenko, A., Chable-Bessia, C., Cantarella, G., Israel, A., Wallach, D. and Courtois, G. (2003) The tumour suppressor CYLD negatively regulates NF-kappaB signalling by deubiquitination. Nature 424, 801-805.
57 Ikeda, F. and Dikic, I. (2006) CYLD in ubiquitin signaling and tumor pathogenesis. Cell 125, 643-645.

58 Massoumi, R., Chmielarska, K., Hennecke, K., Pfeifer, A. and Fassler, R. (2006) Cyld inhibits tumor cell proliferation by blocking Bcl-3-dependent NF-kappaB signaling. Cell 125, 665677.

59 Bode, A.M. and Dong, Z. (2004) Posttranslational modification of p53 in tumorigenesis. Nat Rev Cancer 4, 793-805.

60 Sherr, C.J. (2004) Principles of tumor suppression. Cell 116, 235-246.

61 Brooks, C.L. and Gu, W. (2006) p53 ubiquitination: $\mathrm{Mdm} 2$ and beyond. $\mathrm{Mol}$ Cell 21, 307-315.

62 Oliner, J.D., Kinzler, K.W., Meltzer, P.S., George, D.L. and Vogelstein, B. (1992) Amplification of a gene encoding a p53associated protein in human sarcomas. Nature 358, 80-83.

63 Leach, F.S., Tokino, T., Meltzer, P.S., Burrel, M., Oliner, J.D., Smith, S., Hill, D.E., Sidransky, D., Kinzler, K.W. and Vogelstein, B. (1993) p53 mutation and Mdm2 amplification in human soft tissue sarcomas. Cancer Res 53, 2231-2234.

$64 \mathrm{Li}$, M., Brooks, C.L., Kon, N. and Gu, W. (2004) A dynamic role of HAUSP in the p53-Mdm2 pathway. Mol Cell 13, 879-886.

65 Li, M., Chen, D., Shiloh, A., Luo, J., Nikolaev, A.Y., Qin, J. and Gu, W. (2002) Deubiquitination of p53 by HAUSP is an important pathway for p53 stabilization. Nature 416, 648-653.

66 Cummins, J.M., Rago, C., Kohli, M., Kinzler, K.W., Lengauer, C. and Vogelstein, B. (2004) Tumour suppression: disruption of HAUSP gene stabilizes p53. Nature 428, 1 p following 486.

67 Masuya, D., Huang, C., Liu, D., Nakashima, T., Yokomise, H., Ueno, M. Nakashima, N. and Sumitomo, S. (2006) The HAUSP gene plays an important role in non-small cell lung carcinogenesis through p53-dependent pathways. $J$ Pathol 208, 724-732.

68 Venkitaraman, A.R. (2004) Tracing the network connecting BRCA and Fanconi 
anaemia proteins. Nat Rev Cancer 4, 266-276.

69 Irminger-Finger, I. and Jefford, C.E. (2006) Is there more to BARD1 than BRCA1? Nat Rev Cancer 6, 382-391.

70 Starita, L.M., Machida, Y., Sankaran, S., Elias, J.E., Griffin, K., Schlegel, B.P., Gygi, S.P. and Parvin, J.D. (2004) BRCA1-dependent ubiquitination of gamma-tubulin regulates centrosome number. Mol Cell Biol 24, 8457-8466.

71 D'andrea, A.D. and Grompe, M. (2003) The Fanconi anaemia/BRCA pathway. Nat Rev Cancer 3, 23-34.

72 Gregory, R.C., Taniguchi, T. and D'andrea, A.D. (2003) Regulation of the Fanconi anemia pathway by monoubiquitination. Semin Cancer Biol 13, 77-82.

73 Vandenberg, C.J., Gergely, F., Ong, C.Y., Pace, P., Mallery, D.L., Hiom, K. and Patel, K.J. (2003) BRCA1independent ubiquitination of FANCD2. Mol Cell 12, 247-254.

74 Meetei, A.R., de Winter, J.P., Medhurst, A.L., Wallisch, M., Waisfisz, Q., Van De Vrugt, H.J., Oostra, A.B., Yan, Z., Ling, C., Bishop, C.E., et al. (2003) A novel ubiquitin ligase is deficient in Fanconi anemia. Nat Genet 35, 165170.

75 Nijman, S.M., Huang, T.T., Dirac, A.M., Brummelkamp, T.R., Kerkhoven, R.M., D'andrea, A.D. and Bernards, R. (2005) The deubiquitinating enzyme USP1 regulates the Fanconi anemia pathway. Mol Cell 17, 331-339.

76 Lehmann, A.R. (2006) Translesion synthesis in mammalian cells. Exp Cell Res 312, 2673-2676.

77 Lehmann, A.R. (2005) Replication of damaged DNA by translesion synthesis in human cells. FEBS Lett 579, 873-876.

78 Kaelin, W.G., Jr. (2002) Molecular basis of the VHL hereditary cancer syndrome. Nat Rev Cancer 2, 673-682.

79 Pause, A., Lee, S., Worrell, R.A., Chen, D.Y., Burgess, W.H., Linehan, W.M. and Klausner, R.D. (1997) The von Hippel-Lindau tumor-suppressor gene product forms a stable complex with human CUL-2, a member of the Cdc53 family of proteins. Proc Natl Acad Sci USA 94, 2156-2161.

80 Cockman, M.E., Masson, N., Mole, D.R., Jaakkola, P., Chang, G.W., Clifford, S.C., Maher, E.R., Pugh, C.W., Ratcliffe, P.J. and Maxwell, P.H. (2000) Hypoxia inducible factor-alpha binding and ubiquitylation by the von Hippel-Lindau tumor suppressor protein. J Biol Chem 275, 25733-25741.

81 Blume-jensen, P. and Hunter, T. (2001) Oncogenic kinase signalling. Nature 411, 355-365.

82 Dikic, I. and Giordano, S. (2003) Negative receptor signalling. Curr Opin Cell Biol 15, 128-135.

83 Peschard, P. and Park, M. (2003) Escape from Cbl-mediated downregulation: a recurrent theme for oncogenic deregulation of receptor tyrosine kinases. Cancer Cell 3, 519-523.

84 Bache, K.G., Slagsvold, T. and Stenmark, H. (2004) Defective downregulation of receptor tyrosine kinases in cancer. EMBO J 23, 2707-2712.

85 Mosesson, Y., Shtiegman, K., Katz, M., Zwang, Y., Vereb, G., Szollosi, J. and Yarden, Y. (2003) Endocytosis of receptor tyrosine kinases is driven by monoubiquitylation, not polyubiquitylation. J Biol Chem 278, 21323-21326.

86 Citri, A. and Yarden, Y. (2006) EGFERBB signalling: towards the systems level. Nat Rev Mol Cell Biol 7, 505-516.

87 Sebastian, S., Settleman, J., Reshkin, S.J., Azzariti, A., Bellizzi, A. and Paradiso, A. (2006) The complexity of targeting EGFR signalling in cancer: from expression to turnover. Biochim Biophys Acta 1766, 120-139.

88 Yarden, Y. and Sliwkowski, M.X. (2001) Untangling the ErbB signalling network. Nat Rev Mol Cell Biol 2, 127-137.

89 Lenferink, A.E., Pinkas-Kramarski, R., Van de Poll, M.L., Van Vugt, M.J., Klapper, L.N., Tzahar, E., Waterman, H., Sela, M., Van Zoelen, E.J. and Yarden, Y. (1998) Differential endocytic routing of homo- and hetero-dimeric ErbB tyrosine kinases confers signaling superiority to receptor heterodimers. EMBO J 17, 3385-3397. 
90 Muthuswamy, S.K., Gilman, M. and Brugge, J.S. (1999) Controlled dimerization of ErbB receptors provides evidence for differential signaling by homo- and heterodimers. Mol Cell Biol 19, 6845-6857.

91 Slagsvold, T., Pattni, K., Malerod, L. and Stenmark, H. (2006) Endosomal and non-endosomal functions of ESCRT proteins. Trends Cell Biol 16, 317-326.

$92 \mathrm{Xu}$, Z., Liang, L., Wang, H., Li, T. and Zhao, M. (2003) HCRP1, a novel gene that is downregulated in hepatocellular carcinoma, encodes a growth-inhibitory protein. Biochem Biophys Res Commun 311, 1057-1066.

93 Lee, M.P. and Feinberg, A.P. (1997) Aberrant splicing but not mutations of TSG101 in human breast cancer. Cancer Res 57, 3131-3134.

94 Hariharan, I.K. and Bilder, D. (2006) Regulation of imaginal disc growth by tumor-suppressor genes in Drosophila. Annu Rev Genet 40, 385361.

95 Moberg, K.H., Schelble, S., Burdick, S.K. and Hariharan, I.K. (2005) Mutations in erupted, the Drosophila ortholog of mammalian tumor susceptibility gene 101, elicit non-cellautonomous overgrowth. Dev Cell 9, 699-710.

96 Thompson, B.J., Mathieu, J., Sung, H.H., Loeser, E., Rorth, P. and Cohen, S.M. (2005) Tumor suppressor properties of the ESCRT-II complex component Vps25 in Drosophila. Dev Cell 9, 711-720.

97 Herz, H.M., Chen, Z., Scherr, H., Lackey, M., Bolduc, C. and Bergmann, A. (2006) vps25 mosaics display non-autonomous cell survival and overgrowth, and autonomous apoptosis. Development 133, 1871-1880.

98 Vaccari, T. and Bilder, D. (2005) The Drosophila tumor suppressor vps25 prevents nonautonomous overproliferation by regulating notch trafficking. Dev Cell 9, 687-698.

99 Nalepa, G., Rolfe, M. and Harper, J.W. (2006) Drug discovery in the ubiquitinproteasome system. Nat Rev Drug Discov 5, 596-613.

100 Adams, J. (2004) The development of proteasome inhibitors as anticancer drugs. Cancer Cell 5, 417-421.

101 Richardson, P.G., Mitsiades, C., Hideshima, T. and anderson, K.C. (2005) Proteasome inhibition in the treatment of cancer. Cell Cycle 4, 290-296.

102 Klapper, L.N., Waterman, H., Sela, M. and Yarden, Y. (2000) Tumor-inhibitory antibodies to HER-2/ErbB-2 may act by recruiting $\mathrm{c}-\mathrm{Cbl}$ and enhancing ubiquitination of HER-2. Cancer Res $\mathbf{6 0}$, 3384-3388.

103 Verma, R., Peters, N.R., D’Onofrio, M., Tochtrop, G.P., Sakamoto, K.M., Varadan, R., Zhang, M., Coffino, P., Fushman, D., Deshaies, R.J. and King, R.W. (2004) Ubistatins inhibit proteasome-dependent degradation by binding the ubiquitin chain. Science 306, 117-120. 